\title{
Pamidronato endovenoso en espondilitis anquilosante refractaria a antiinflamatorios no esteroideos (AINES) y sulfasalazina
}

\author{
Augusto García-Poma ${ }^{1,2}$, Manuel Montero-Jauregui ${ }^{2}$, Henry Terrazas ${ }^{1,2}$, \\ Tatiana Miraval ${ }^{2}$, Felipe Becerra ${ }^{1,2}$, María I Segami ${ }^{1,2}$
}

Resumen

Palabras clave
Objetivo: Evaluar la terapia con pamidronato, en pacientes con espondilitis anquilosante (EA) activa, con respuesta subóptima o falla a los antiinflamatorios no-esteroideos (AINES) y sulfasalazina. Diseño: Estudio clínico comparativo. Lugar: Hospital Nacional Edgardo Rebagliati Martins, EsSalud, Lima, Perú. Participantes: Pacientes con espondilitis anquilosante. Intervenciones. Se incluyó 9 pacientes con EA(6 varones), con enfermedad activa (BASDAI $\geq 4$ ), actividad axial y falta de respuesta a los Aines y sulfasalazina a dosis $\mathrm{de} 3 \mathrm{~g} / \mathrm{d}$. Todos los pacientes recibieron $60 \mathrm{mg}$ de pamidronato mensual, en infusión endovenosa, durante 6 meses, $y$ continuaron tomando AINESy sulfasalazina. La mejoría clínica fue evaluada usando el Asas 20. En forma secundaria se evaluó el ASAS 40, BASDAI50, BASDAI, BASFIY BASMI, a las 24 y 48 semanas (32 a 86 semanas). La diferencia entre el índice de pre y postratamiento fue evaluada usando la prueba de Wilcoxon. Principales medidas de resultados: Evaluación del ASAS 20. Resultados. El 67\% alcanzó un Asas 20 a las 24 semanas y 78\% a las 48 semanas; 33,3\% y 55,6\% tuvieron ASAS 40 y 33,3\%; y 44,4\% alcanzó BASDAI 50 a las 24 y 48 semanas, respectivamente. Un paciente recayó a la semana 20 . Tres pacientes (33,3\%) permanecieron sin cambios. A las 24 y 48 semanas, la media de BASDAI disminuyó en 45,1\% ( $p=0,007)$ y en $52,1 \%$ $(p=0,01)$, la media de BASFI en $38,2 \%(p=0,007)$ y en $52,3 \%$ ( $p=0,007)$, y la media de BASMI en $39,2 \%(p=0,01)$ y 39,2\% ( $p=0,01)$, respectivamente. Los eventos adversos no fueron importantes con esta terapia. Conclusiones. El tratamiento con pamidronato demostró ser efectivo en este grupo de pacientes con EA, refractaria a AINES y a sulfasalazina.

Espondilitis anquilosante; pamidronato; agentes antiinflamatorios no esteroides; sulfasalozina.
Intravenous pamidronate in refractory ankylosing spondylitis

Abstract

Objective: To determine the response of an aminobisphosphonate (pamidronate) in patients with ankylosing spondylitis (AS) who had suboptimal or no response to nonsteroidal anti-inflammatory drugs (NSAIDs) and sulfasalazine. Design: Comparative clinical study. Setting: Hospital Nacional Edgardo Rebagliati Martins, EsSalud, Lima, Peru. Participants: Patients with ankylosing spondylitis. Intervenciones: Nine patients with AS (6 males), with active disease [BASDAI $\geq 4]$ and no response to NSAIDs and sulfasalazine up to $3 g /$ day entered the study. All patients received monthly infusions of $60 \mathrm{mg}$ of pamidronate for 6 months and remained taking NSAID and sulfasalazine. Clinical improvement was evaluated using the Assessments in Ankylosing Spondylitis

1 Universidad Nacional Mayor de San Marcos. Lima, Perú.

Departamento de Enfermedades Sistémicas. Hospital Nacional Edgardo Rebagliati Martins, EsSalud. Lima, Perú.
20(ASAS 20). Secondary evaluations included ASAS 40, BASDAI 50, BASDAI, BASFI, and BASMI at 24 weeks and at last observation [48 weeks (32 to 86 weeks)]. Differences between pre and post treatment distributions of all continuous indices were evaluated using the Wilcoxon signed rank test. Main outcome measures: Evaluated of ASAS 20. Results: Sixty-seven percent achieved ASAS 20 at 24 weeks and $78 \%$ at 48 weeks; $33,3 \%$ and 55,6\% achieved ASAS 40 at 24 and 48 weeks, respectively, and 33,3\% and 44,4\% achieved BASDAI 50 at weeks 24 and 48, respectively. One patient relapsed at week 20 . In three patients $(33,3 \%)$ the scores remained unchanged. At weeks 24 and 48 mean BASDAI decreased by $45,1 \%(p=0,007)$ and by $52,1 \%$ $(p=0,01)$, mean BASFI decreased by 38,2\% $(p=0,007)$ and by $52,3 \%(p=0.007)$, and mean BASMI decreased by 39,2\% $(p=0,01)$ and $39,2 \%(p=0,01)$, respectively. There were no significant adverse events with this therapy. Conclusions: Our data provide further evidence of pamidronate therapy effectiveness in patients with AS who are refractory to NSAIDs and sulfasalazine.

Key words: Spondylitis, ankylosing; pamidronate; antiinflammatory agents; non-steroidal; sulfasalazine. 


\section{INTRODUCCIÓN}

La espondilitis anquilosante (EA) es una enfermedad inflamatoria crónica, discapacitante, con deterioro progresivo de la función física, la calidad de vida y disminución de la masa ósea ${ }^{(1-4)}$. Inicialmente, compromete el esqueleto axial, generalmente las articulaciones sacroilíacas, y las articulaciones periféricas hasta en $47 \%\left({ }^{5,6}\right)$. Se asocia al antígeno HLA-B27, en $90 \%$ de los casos $\left({ }^{4,7-9}\right)$ La prevalencia se estima entre 0,1 y $1,4 \%$ de la población adulta, afectando predominantemente a los hombres, tres veces más que a las mujeres, generalmente entre la segunda y tercera década de la vida, con presentación de los primeros síntomas entre los 15 y 40 años, hasta en $90 \%$ de casos $(2,5,10,11)$.

El deterioro progresivo de la función física y la calidad de vida en los pacientes con EA se asocia a actividad de la enfermedad $(2,12,13)$ y los costos de salud, principalmente costos directos, tales como servicios médicos y tratamiento de la enfermedad, que influyen en la calidad de vida del paciente $\left({ }^{10}\right)$. El tratamiento de primera línea en los pacientes con EA consiste en los antiinflamatorios no esteroideos (AINES) y la fisioterapia $\left({ }^{8,14,15}\right)$. Sin embargo, se cuenta con drogas antirreumáticas modificadoras de la enfermedad (DARMES), bifosfonatos y talidomida, como alternativas en algunos reportes de ensayos $\left({ }^{16}\right)$, y la terapia biológica (anti-TNF- $\alpha$ ), mundialmente usadas como tratamiento de la EA $\left({ }^{8,14,15}\right)$. El uso de la terapia biológica viene dando buenos resultados, pero su alto costo y el riesgo de complicaciones infecciosas -como la tuberculosis- $\left({ }^{17}\right)$ limitan su uso.

Se ha utilizado los bifosfonatos, en particular el pamidronato, en el tratamiento de pacientes con EA activa resistente a los AINES $\left({ }^{18-23}\right)$. El pamidronato ha sido usado no solo en el manejo de la osteoporosis y otros desórdenes del metabolismo óseo ${ }^{24-}$ ${ }^{26}$ ), por su efecto inhibitorio del osteoclasto, la mejoría de la densidad mineral ósea y la prevención de las fracturas $\left(^{3,4}\right)$, sino además por su efecto antiinflamatorio (suprimen las citoquinas proinflamatorias, tales como IL1, IL-6 y TNF) ${ }^{\left({ }^{27}\right)}$. Se ha usado en pacientes con EA con resultados beneficiosos, luego de su administración endovenosa (IV) (18${ }^{20}$ ). Maksymowych WP y col, en una población canadiense de 84 pacientes con EA, comunicaron disminución significativa del BASDAI, BASFI Y BASMI, después de 6 meses de tratamiento, con dosis mensuales de $60 \mathrm{mg}$ de pamidronato IV. Además, $63,4 \%$ de los pacientes informó una reducción de alrededor de $\geq 25 \%$ del BASDAI, a los 6 meses $\left({ }^{20}\right)$. Sin embargo, otros estudios encontraron modestos resultados, después de la administración de pamidronato $\left({ }^{21-23}\right)$. El uso de pamidronato IV se asocia a la presencia transitoria de artralgias, mialgias y fiebre, luego de la primera infusión $\left({ }^{182}\right)$.

Nosotros realizamos este estudio para evaluar el uso de pamidronato en pacientes con EA, con persistencia de actividad de enfermedad axial, a pesar del uso de AINES y sulfasalazina ( $\sin$ beneficios significativos a nivel axial, pero sí en articulaciones periféricas) $\left({ }^{28}\right)$. La dosis que usamos fue similar a la informada en estudios ya publicados y que mostraron resultados significativos $\left({ }^{20}\right)$.

\section{MATERIALES Y MÉTODOS}

Se incluyó 9 pacientes mayores de 18 años, con diagnóstico de EA (6 hombres y 3 mujeres), según los criterios modificados de Nueva York (1984) $\left.{ }^{(29}\right)$, con enfermedad activa (Bath AS Disease Activity Index [BASDAI] $\geq 4)\left({ }^{30}\right)$, principalmente actividad axial y falta de respuesta a los AINES y sulfasalazina, a dosis de $3 \mathrm{~g} / \mathrm{d}$, en el periodo comprendido entre enero de 2003 y marzo de 2006, hospitalizados en el Servicio de Reumatología del Hospital Nacional Edgardo Rebagliati Martins, Lima, Perú. Todos los pacientes recibieron mensualmente $60 \mathrm{mg}$ de pamidronato en infusión endovenosa, administrados en $600 \mathrm{~mL}$ de dextrosa, durante 6 
horas (para disminuir las artralgias y mialgias postinfusión $\left({ }^{18-23}\right)$ ), previo control de niveles de calcio sérico (para prevenir la posible hipocalcemia $\left({ }^{31}\right)$ ). Los pacientes continuaron tomando Aines y sulfasalazina.

La mejoría clínica fue evaluada mediante el ASAS 20 (Assessment In Ankylosing Spondylitis), definida como la mejoría de un cambio $\geq 20 \%$ y mejoría absoluta de $\geq 10 \mathrm{~mm}$ sobre una escala de 0 a 100 , en tres de los cuatro dominios, y ausencia de empeoramiento (deterioro $\geq 20 \% \mathrm{y} \geq 10 \mathrm{~mm}$, sobre una escala de 0 a 100), en el cuarto dominio. Los 4 dominios incluyen: evaluación global del paciente, dolor axial, función (evaluado por el BASFI [índice funcional de EA]) e inflamación (evaluado por la media de dos puntajes del BASDAI, relacionados a rigidez matutina $5^{\mathrm{a}}$ y $6^{\mathrm{a}}$ preguntas) ${ }^{32}$ ). En forma secundaria, se evaluó el ASAS 40 (definida como la mejoría $\geq 40 \%$ y $\geq 20 \mathrm{~mm}$ sobre una escala de 0 a 100 , en al menos tres de cuatro dominios, y no empeoramiento del cuarto dominio) $\left({ }^{33,34}\right)$, el índice de actividad de la enfermedad de EA (Bath AS Disease Activity Index, BASDAI) $\left({ }^{30}\right)$, el índice funcional de EA (Bath AS Functional Index, BASFI) $\left({ }^{35}\right)$, el índice metrológico de EA (Bath AS Metrology Index, BASMI) ( ${ }^{36}$ ) y el BASDAI 50 (definida como la mejoría del $50 \%$ de la basal o una mejoría de dos unidades sobre una escala de 10 unidades del BASDAI) $\left.{ }^{(37}\right)$, a las 24 y 48 semanas. Se realizó la evaluación clínica basal antes de la primera infusión y dos controles finales: el primero al término del tratamiento, a las 24 semanas (6 meses), y el segundo a las 48 semanas (32 a 86 semanas).

Se registró las características demográficas y clínicas de todos los pacientes incluidos en el estudio, así como los datos clinimétricos de actividad de enfermedad, índice funcional y de movilidad en los pacientes con EA, mediante el BASDAI, BASFI y BASMI, y datos de mejoría clínica evaluados por el ASAS 20, ASAS 40 y BASDAI 50. Se usó el chi-cuadrado para las variables categóricas. Se evaluó la diferencia entre el índice pre y postratamiento, con la prueba de Wilcoxon. Para el análisis, se usó el paquete estadístico Stata 8.2. Se consideró valor estadísticamente significativo $p<0,05$.

\section{RESULTADOS}

De los 10 pacientes que iniciaron tratamiento con pamidronato, solo se incluyó en el presente estudio 9 pacientes, que completaron el tratamiento de 6 meses.

La edad promedio fue $36 \pm 15$ años, el $67 \%$ de sexo masculino; $33 \%$ tuvo HLAB27 positivo y el promedio de la duración de enfermedad fue $19 \pm 10$ años (Tabla 1).

Sesenta y siete por ciento de los pacientes alcanzaron un ASAS 20 a las 24 semanas y el $78 \%$ a las 48 semanas. Porcentajes de $33,3 \%$ y $55,6 \%$ alcanzaron un ASAS 40 a las 24 y 48 semanas, respectivamente; $33,3 \%$, un BASDAI-50 a las 24 semanas y $44,4 \%$, a las 48 semanas (Figura 1). Además, se observó BASDAI 70 en $11,1 \%$ y $22,2 \%$, a las 24 y 48 semanas (datos no mostrados).

Un paciente recayó a la semana 20, después de haber culminado el curso de 6 infusiones. En tres pacientes $(33,3 \%)$ no hubo mejoría (fueron pacientes con una media de 28,3 años de enfermedad vs. 14 años en los pacientes que sí respondieron).

En la mejoría de los parámetros clinimétricos, la media del puntaje de la actividad de la enfermedad del EA (BASDAI) disminuyó en $45,1 \%(p=0,007)$

Tabla 1. Características de los pacientes con espondilitis anquilosante.

\begin{tabular}{lc}
\hline Pacientes (n) & 9 \\
Edad (años) & $36 \pm 15$ \\
Sexo: masculino & $67 \%$ \\
Duración de la enfermedad (años) & $19 \pm 10$ \\
HLA-B27 positivo & $33 \%$ \\
\hline
\end{tabular}

Variables continuas son presentados como media $\pm D E$ y categóricas como frecuencias y porcentajes. 


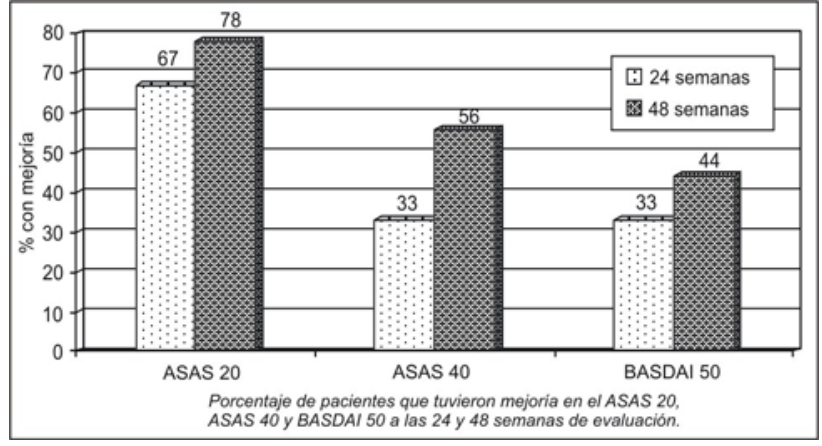

Figura 1. Evaluación de la mejoría clínica y de la actividad de la enfermedad en los pacientes con EA por ASAS 20, ASAS 40 y BASDAI 50.

y en $52,1 \%(p=0,01)$, a las 24 y 48 semanas; la media del índice funcional del EA (BASFI) disminuyó en $38,2 \%(p=0,007)$ y en $52,3 \%(p=0,007)$, y la media del índice metrológico del EA (BASMI) disminuyó en $39,2 \%(p=0,01)$ y $39,2 \%(p=0,01)$, a las 24 y 48 semanas (Tabla 2 y Figura 2).

Tabla 2. Resultados de la evaluación clinimétricas basal, a las 24 y 48 semanas, en pacientes con espondilitis anquilosante, quienes recibieron infusión IV de pamidronato.

\begin{tabular}{|c|c|c|c|c|c|c|c|}
\hline \multirow{2}{*}{\multicolumn{2}{|c|}{$\begin{array}{c}\text { Basal } \\
\text { media } \pm \mathrm{DE}\end{array}$}} & \multicolumn{3}{|c|}{24 semanas } & \multicolumn{3}{|c|}{48 semanas } \\
\hline & & media $\pm \mathrm{DF}$ & $\downarrow \%$ & $p$ & media $\pm \mathrm{DE}$ & $\downarrow \%$ & $p$ \\
\hline AS & I 7, & $3,9 \pm 2,2$ & 45,1 & 0,007 & $3,4 \pm 2,7$ & 52,1 & 0,01 \\
\hline BASFI & $8,0 \pm 1,4$ & $4,9 \pm 2,7$ & 38,2 & 0,007 & $3,8 \pm 3,7$ & 52,3 & 0,007 \\
\hline BASMI & $5,7 \pm 1,9$ & $3,4 \pm 2,1$ & 39,2 & 0,01 & $3,4 \pm 2,7$ & 39,2 & 0,01 \\
\hline
\end{tabular}

No se presentaron eventos adversos de importancia en los pacientes que completaron el tratamiento, a excepción del paciente que fue excluido del estudio por presentar artralgias severas a la primera infusión. Durante el estudio, uno de los pacientes presentó dolor de una cadera, mejorando notoriamente con corticoide intraarticular.

\section{DISCUSIÓN}

El uso mensual de $60 \mathrm{mg}$ de pamidronato endovenoso, por seis meses continuos, en pacientes con EA con actividad axial refractaria a los AINES y sulfasalazina, fue efectivo.
En la evaluación clínica del ASAS 20, se observó resultados muy aceptables a las 24 y 48 semanas del estudio $(67 \%$ y $78 \%)$, a diferencia de lo encontrado en otros estudios $\left({ }^{21,23}\right)$, con resultados modestos a los 6 meses de estudio. El 33,3\% de los pacientes alcanzó un ASAS 40 a las 24 semanas y un $55,6 \%$, a las 48 semanas, a diferencia de otro estudio $\left({ }^{23}\right)$, en donde no se observó algún ASAS 40 al término del estudio.

En la mejoría de la actividad de la enfermedad, el $33 \%$ alcanzó BASDAI-50 a las 24 semanas (6 meses) y $44,4 \%$, a las 48 semanas. En el estudio doble ciego controlado de Maksymowych WP y col $\left({ }^{20}\right)$, en 84 pacientes con EA, se alcanzó un BASDAI 50 en $39 \%$ de los pacientes que recibieron $60 \mathrm{mg}$ mensuales de pamidronato vs. $16,3 \%$ en los que recibieron $10 \mathrm{mg}$ mensuales por 6 meses. Mientras que, otro estudio $\left({ }^{21}\right)$ observó mejoría solo en $1 / 12(8,3 \%)$, después de los 3 meses, y en $3 / 9(33,3 \%)$, a los 6 meses. En nuestro estudio, observamos mejoría del BASDAI 70 en $11,1 \%$, a las 24 semanas, y en $22,2 \%$, a las 48 semanas. En el estudio antes mencionado, se observó un BASDAI 70 en $17 \%$, en el grupo de pacientes con $60 \mathrm{mg}$ vs. $4,7 \%$ en aquellos con $10 \mathrm{mg}$, a los 6 meses de estudio $\left({ }^{20}\right)$.

En la evaluación de los parámetros clinimétricos BASDAI, BASFI y BASMI, la media del puntaje de la actividad de la enfermedad del EA (BASDAI) disminuyó en

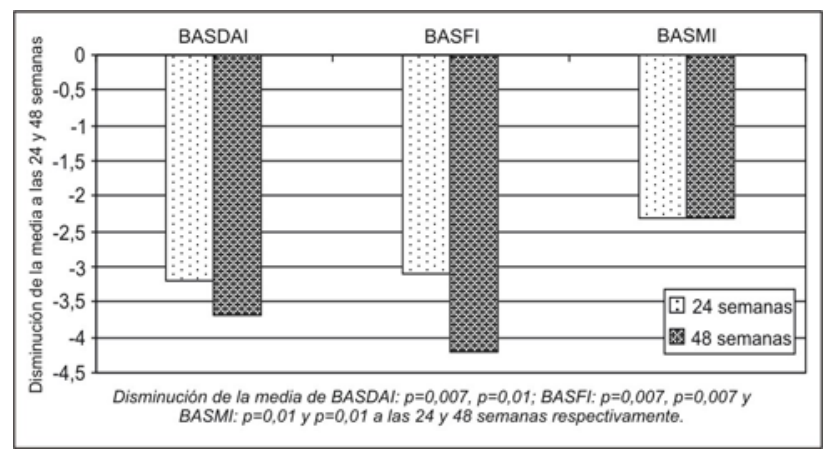

Figura 2. Disminución del BASDAI, BASFI y BASMI a las 24 y 48 semanas. 
$45,1 \%(p=0,007)$ y en $52,1 \%(p=0,01)$, a las 24 y 48 semanas, respectivamente. Esto fue similar a otros estudios, $\left({ }^{18,20,22}\right)$ en donde se observó mejoría significativa en la reducción de la media de BASDAI. Mientras que, en un estudio ${ }^{21}$ ) se observó una mejoría significativa de la media de BASDAI a los 3 meses y no después de los 6 meses de estudio. La media del índice funcional del EA (BASFI) disminuyó significativamente a las 24 y 48 semanas, similar a lo observado en otros estudios a los tres y 6 meses $\left({ }^{19,20}\right)$. En tanto que, en otros dos estudios $\left({ }^{21,23}\right)$ no se encontró mejoría estadísticamente significativa. La media del índice metrológico del EA (BASMI) disminuyó significativamente, similar a lo hallado en otros estudios $\left({ }^{18,20}\right)$ y a diferencia de otros, en donde no se observó resultados significativos $\left({ }^{21,22}\right)$

Este estudio tiene limitaciones. Es un estudio abierto, con un número pequeño de pacientes. Pero, todos tuvieron actividad importante de enfermedad y compromiso funcional, a diferencia de otros reportes (75\% de los pacientes tenía anquílosis completa de las sacroilíacas) $\left({ }^{21}\right)$. En nuestro estudio, los pacientes incluidos completaron el periodo de seis meses de tratamiento, a diferencia de otros estudios en que alrededor de $20 \%$ a $30 \%$ de los pacientes no completaron el tratamiento, ya sea por efectos adversos, ineficacia o retiro $\left.{ }^{21-23}\right)$. Además, se observó mejores resultados a las 48 semanas que a las 24 semanas. (Figura 1 y 2, Tabla 2). Se conoce de otros estudios en los que el efecto del pamidronato es tardío (diferencia no significativa en los parámetros clinimétricos a los 3 meses, pero sí a los 6 meses) y dosis dependiente $\left({ }^{20}\right)$.

Es importante tener en cuenta el costo del cuidado de la salud en este tipo de enfermedades crónicas. El costo de la administración de pamidronato por seis meses de infusión IV es aproximadamente 850 dólares, en nuestro medio, que es mucho menor con relación a terapia biológica (antiTNF- $\alpha$ ) $\left.{ }^{38}\right)$, por lo que podría constituirse en una terapia alternativa, por su efecto antiinflamatorio en pacientes con EA con actividad axial refractaria a los AINES y/o DARMES, en países en vías de desarrollo, como el nuestro.

En conclusión, el tratamiento con 6 dosis mensuales de $60 \mathrm{mg}$ de pamidronato demostró ser efectivo en un grupo de pacientes con EA, con gran actividad de enfermedad axial refractaria al tratamiento con AINES y sulfasalazina, sugiriendo su uso como tratamiento alternativo.

\section{AGRADECIMIENTO}

Al Dr. Carlos G Grijalva, por el apoyo en el desarrollo del análisis estadístico de los datos, y a la Dra. Cecilia P. Chung por la revisión crítica del manuscrito.

\section{REFERENCIAS BIBLIOGRÁFICAS}

1. Ozgul A, Peker F, Taskaynatan MA, Tan AK, Dincer K, Kalyon TA. Effect of ankylosing spondylitis on health-related quality of life and different aspects of social life in young patients. Clin Rheumatol. 2006;25:168-74.

2. Davis JC, van der Heijde D, Dougados M, Woolley JM. Reductions in health-related quality of life in patients with ankylosing spondylitis and improvements with etanercept therapy. Arthritis Rheum. 2005;53:494-501.

3. Meirelles ES, Borelli A, Camargo OP. Influence of disease activity and chronicity on ankylosing spondylitis bone mass loss. Clin Rheumatol. 1999;18:364-8.

4. El Maghraoui A. Osteoporosis and ankylosing spondylitis. Joint Bone Spine. 2004;71(4):291-5.

5. Sieper J, Braun J, Rudwaleit M, Boonen A, Zink A. Ankylosing spondylitis: an overview. Ann Rheum Dis. 2002;61 Suppl 3:iii8-18.

6. Brophy S, Mackay K, Al-Saidi A, Taylor G, Calin A. The natural history of ankylosing spondylitis as defined by radiological progression. J Rheumatol. 2002;29:1236-43.

7. Khan MA. Update on spondyloarthropathies. Ann Intern Med. 2002;136:896-907.

8. Anandarajah A, Ritchlin CT. Treatment update on spondyloarthropathy. Curr Opin Rheumatol. 2005;17:247-56.

9. van der Linden SM, Valkenburg HA, de Jongh BM, Cats A. The risk of developing ankylosing spondylitis in HLAB27 positive individuals. A comparison of relatives of spondylitis patients with the general population. Arthritis Rheum. 1984;27:241-9.

10. Boonen A, van der Linden SM. The burden of ankylosing spondylitis. J Rheumatol Suppl. 2006;78:4-11.

11. Bakland G, Nossent HC, Gran JT. Incidence and prevalence of ankylosing spondylitis in Northern Norway. Arthritis Rheum. 2005;53:850-5. 
12. Dagfinrud H, Kjeken I, Mowinckel P, Hagen KB, Kvien TK. Impact of functional impairment in ankylosing spondylitis: impairment, activity limitation, and participation restrictions. J Rheumatol. 2005;32:516-23.

13. Bostan EE, Borman P, Bodur H, Barca N. Functional disability and quality of life in patients with ankylosing spondylitis. Rheumatol Int. 2003;23:121-6.

14. Scalapino KJ, Davis JC Jr. The treatment of ankylosing spondylitis. Clin Exp Med. 2003;2:159-65.

15. Reveille JD, Arnett FC. Spondyloarthritis: update on pathogenesis and management. Am J Med. 2005;118:592-603.

16. Davis JC Jr, Huang F, Maksymowych W. New therapies for ankylosing spondylitis: etanercept, thalidomide, and pamidronate. Rheum Dis Clin North Am. 2003;29(3):481-94.

17. Braun J, Sieper J. Biological therapies in the spondyloarthritides-the current state. Rheumatology. 2004;43:1072-84.

18. Maksymowych WP, Jhangri GS, Leclercq S, Skeith K, Yan A, Russell AS. An open study of pamidronate in the treatment of refractory ankylosing spondylitis. J Rheumatol. 1998;25:714-7.

19. Maksymowych WP, Lambert R, Jhangri GS, Leclercq S, Chiu P, Wong B, et al. Clinical and radiological amelioration of refractory peripheral spondyloarthritis by pulse intravenous pamidronate therapy. J Rheumatol. 2001;28:144-55.

20. Maksymowych WP, Jhangri GS, Fitzgerald AA, LeClercq S, Chiu P, Yan A, et al. A six-month randomized, controlled, double-blind, dose-response comparison of intravenous pamidronate $(60 \mathrm{mg}$ versus $10 \mathrm{mg}$ ) in the treatment of nonsteroidal antiinflammatory drug-refractory ankylosing spondylitis. Arthritis Rheum. 2002;46:766-73.

21. Haibel H, Brandt J, Rudwaleit M, Soerensen H, Sieper J, Braun J. Treatment of active ankylosing spondylitis with pamidronate. Rheumatology. 2003;42:1018-20.

22. Cairns AP, Wright SA, Taggart AJ, Coward SM, Wright GD. An open study of pulse pamidronate treatment in severe ankylosing spondylitis, and its effect on biochemical markers of bone turnover. Ann Rheum Dis. 2005;64:338-9.

23. Grover R, Shankar S, Aneja R, Marwaha V, Gupta R, Kumar A. Treatment of ankylosing spondylitis with pamidronate: an open label study. Ann Rheum Dis. 2006;65:688-9.

24. Bembi B, Parma A, Bottega M, Ceschel S, Zanatta M, Martini $\mathrm{C}$, et al. Intravenous pamidronate treatment in osteogenesis imperfecta. J Pediatr. 1997;131:622-5.

25. Krieg MA, Seydoux C, Sandini L, Goy JJ, Berguer DG, Thiebaud D, et al. Intravenous pamidronate as treatment for osteoporosis after heart transplantation: a prospective study. Osteoporos Int. 2001;12:112-6.

26. Stokkers PC, Deley M, Van Der Spek M, Verberne HJ, Van Deventer SJ, Hommes DW. Intravenous pamidronate in combination with calcium and vitamin D: highly effective in the treatment of low bone mineral density in inflammatory bowel disease. Scand J Gastroenterol. 2006;41(2):200-4.

27. Pennanen N, Lapinjoki S, Urtti A, Monkkonen J. Effect of liposomal and free bisphosphonates on the IL-1 beta, IL-6 and TNF alpha secretion from RAW 264 cells in vitro. Pharm Res. 1995;12(6):916-22.

28. Clegg DO, Reda DJ, Abdellatif M.Comparison of sulfasalazine and placebo for the treatment of axial and peripheral articular manifestations of the seronegative spondylarthropathies: a Department of Veterans Affairs cooperative study. Arthritis Rheum. 1999;42:2325-9.

29. van der Linden S, Valkenburg HA, Cats A. Evaluation of diagnostic criteria for ankylosing spondylitis. A proposal for modification of the New York criteria. Arthritis Rheum. 1984;27:361-8.

30. Garrett S, Jenkinson T, Kennedy LG, Whitelock H, Gaisford $\mathrm{P}$, Calin A. A new approach to defining disease status in ankylosing spondylitis: the Bath ankylosing spondylitis disease activity index. J Rheumatol. 1994;21:2286-91.

31. Tanvetyanon T, Stiff PJ. Management of the adverse effects associated with intravenous bisphosphonates. Ann Oncol. 2006;17:897-907.

32. Anderson JJ, Baron G, van der Heijde D, Felson DT, Dougados M. Ankylosing spondylitis assessment group preliminary definition of short-term improvement in ankylosing spondylitis. Arthritis Rheum. 2001;44:1876-86.

33. Brandt J, Listing J, Sieper J, Rudwaleit M, van der Heijde D, Braun J. Development and preselection of criteria for short term improvement after anti-TNF alpha treatment in ankylosing spondylitis. Ann Rheum Dis. 2004;63:1438-44.

34. Zochling J, Braun J, van der Heijde D. Assessments in ankylosing spondylitis. Best Pract Res Clin Rheumatol. 2006;20:521-37.

35. Calin A, Garrett S, Whitelock H, Kennedy LG, O'Hea J, Mallorie P, et al. A new approach to defining functional ability in ankylosing spondylitis: the development of the Bath ankylosing spondylitis functional index. J Rheumatol.1994;21:2281-5.

36. Jenkinson TR, Mallorie PA, Whitelock HC, Kennedy LG, Garrett SL, Calin A. Defining spinal mobility in ankylosing spondylitis (AS). The Bath AS metrology index. J Rheumatol. 1994;21:1694-8.).

37. Braun J, Pham T, Sieper J, Davis J, van der Linden S, Dougados $\mathrm{M}$, et al. International ASAS consensus statement for the use of anti-tumour necrosis factor agents in patients with ankylosing spondylitis. Ann Rheum Dis. 2003;62:817-24.

38. Kobelt G, Andlin-Sobocki P, Maksymowych WP. Costs and quality of life of patients with ankylosing spondylitis in Canada. J Rheumatol. 2006;33(2):289-95.

Manuscrito recibido el 25 de enero de 2007 y aceptado para publicación el 06 de febrero de 2007.

Correspondencia:

Augusto García Poma

Departamento de Enfermedades Sistémicas,

Servicio de Reumatología

Hospital Nacional Edgardo Rebagliati Martins

Av. Rebagliati 490. Lima 11, Perú.

Correo-e: agarciapoma@yahoo.es 